\title{
Evaluation of Guarded High-Resistance Hamon Transfer Standards
}

\author{
Dean G. Jarrett, Senior Member, IEEE
}

\begin{abstract}
An improved design for a guarded transfer standard in the resistance range $1 \mathrm{M} \Omega$ to $100 \mathrm{G} \Omega$ is described. Existing transfer standards and their limitations are reviewed along with a description of guard circuit theory. Measurements made to evaluate the effectiveness of guard networks are described and results are reported that verify the guard circuit behavior. Guarded transfer standards have been tested with internal and external guard networks showing the benefits of guarding transfer standards for high-resistance measurements. Interchangeable guard networks are used in the improved transfer standards to ensure complete guarding during all phases of the measurement process thus reducing errors caused by leakages to ground. These improved transfer standards have been developed to support $\mathrm{Na}$ tional Institute of Standards and Technology (NIST) calibration services over the range $10 \mathrm{M} \Omega$ to $1 \mathrm{~T} \Omega$ and to support extension of the calibration service to $10 \mathrm{~T} \Omega$ and $100 \mathrm{~T} \Omega$.
\end{abstract}

Index Terms - Bridge circuits, insulation, measurement standards, resistance measurement, resistors, transfer standards.

\section{INTRODUCTION}

$\mathbf{N}$ ATIONAL Institute of Standards and Technology (NIST)-built transfer standards, or Hamon boxes [1], have been used at NIST for many years to scale from one decade of resistance to another [2]. Most of the NIST-built transfer standards use internal guard networks to reduce errors caused by leakage currents flowing from the main resistor network to ground. A NIST-built guarded Wheatstone bridge and a NIST-built guarded active-arm bridge [3] take full advantage of the guard networks associated with the transfer standards. The largest contributing source of the Type B relative standard uncertainty for the active-arm bridge at 10 $\mathrm{G} \Omega$ and above is the stability of the standards. Only film-type resistance elements [4], which exhibit large drift rates, voltage coefficients, and temperature coefficients, are available at 1 $\mathrm{G} \Omega$ and above for construction of high-resistance transfer standards. Techniques developed at NIST [4] to heat treat and mount film-type resistors have been used to construct these guarded high-resistance transfer standards.

The circuit for a typical high-resistance transfer standard is shown in Fig. 1. Ten resistors of the same nominal value are permanently connected in series. Paralleling fixtures can be used to connect the resistors in parallel or series-parallel configurations [2] generating accurate 100:1 and 10:1 ratios for

Manuscript received July 2, 1998.

The author is with the Electricity Division, Electrical and Electronics Engineering Laboratory, National Institute of Standards and Technology, Technology Administration, U. S. Department of Commerce, Gaithersburg, MD 20899-0001 USA (e-mail: dean.jarrett@nist.gov).

Publisher Item Identifier S 0018-9456(99)03193-9.

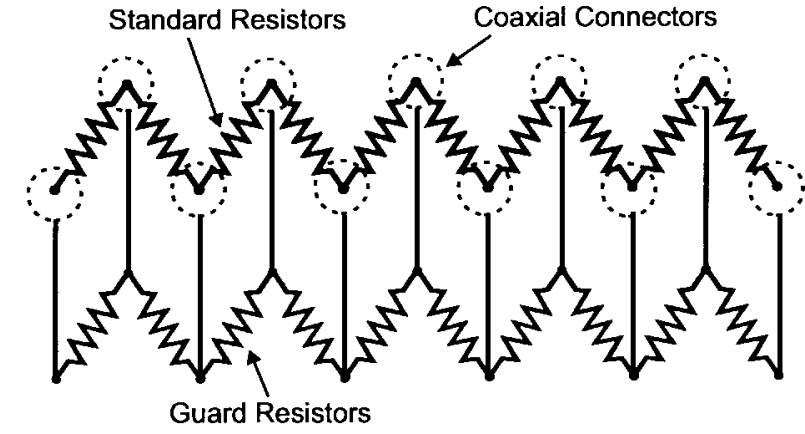

Fig. 1. Guarded transfer standard. Coaxial terminations of each resistor are driven at a guard potential.

scaling to different resistance levels. An internal guard network is shown that drives the coaxial shield of each resistor junction at a guard potential nominally equal to the potential of the main resistor at that point in the network. As long as the guard resistors can drive the shields to the same potential as the inner conductors, very little leakage current flows between the main and guard resistor networks. Any leakage currents to ground flow from the driven guard circuit shields to ground potential, having negligible effect on the main resistor network.

Unfortunately, the fixed value of the guard resistors sometimes makes guarding using the internal guard networks impossible when comparing two transfer standards that have different main-to-guard ratios. The different internal guard resistor networks place the coaxial shields at potentials different than the inner conductors making it impossible to balance the bridge due to excessive leakage currents flowing between the guard and the high input of the bridge null detector. An improved guarded transfer standard has been developed that overcomes some of the problems encountered with guard networks when scaling from one resistance decade to another. Coaxial plug-type connectors are used to provide quick connection of parallel fixtures, series-parallel fixtures, and external guard networks that provide the proper voltages at the coaxial shields to guard the main resistor junctions. The external guard networks allow transfer standards with different internal guard networks to be compared in a guarded bridge circuit with all main resistor junctions fully guarded.

Procedures used to evaluate the guarded transfer standards, circuit theory of the guard network, and measurements made during the evaluation are reported below. The techniques developed here will be used to build additional transfer standards at the $1 \mathrm{~T} \Omega$ level and above to support NIST measurements to $100 \mathrm{~T} \Omega$. 
TABLE I

Series Values for NIST High Resistance Transfer STANDARDS AND INTERNAL GUARD RESISTORS

\begin{tabular}{c|c|c|c}
\hline $\begin{array}{c}\text { Serial } \\
\text { Number }\end{array}$ & $\begin{array}{c}\text { Main } \\
\text { Series } \\
\text { Value }\end{array}$ & $\begin{array}{c}\text { Guard } \\
\text { Series } \\
\text { Value }\end{array}$ & $\begin{array}{c}\text { Ratio } \\
\text { (Main-to- } \\
\text { Guard Ratio) }\end{array}$ \\
\hline $\mathrm{J} / 6$ & $1 \mathrm{M} \Omega$ & $1 \mathrm{M} \Omega$ & $1: 1$ \\
\hline $\mathrm{J} / 7$ & $10 \mathrm{M} \Omega$ & $10 \mathrm{M} \Omega$ & $1: 1$ \\
\hline $\mathrm{J} / 8$ & $100 \mathrm{M} \Omega$ & $1 \mathrm{M} \Omega$ & $100: 1$ \\
\hline $\mathrm{J} / 895$ & $100 \mathrm{M} \Omega$ & $100 \mathrm{M} \Omega$ & $1: 1$ \\
\hline $\mathrm{J} / 9$ & $1 \mathrm{G} \Omega$ & $10 \mathrm{M} \Omega$ & $100: 1$ \\
\hline $\mathrm{C} / 10$ & $10 \mathrm{G} \Omega$ & none & N/A \\
\hline $\mathrm{BS} 10$ & $10 \mathrm{G} \Omega$ & none & N/A \\
\hline $\mathrm{C} 1098$ & $10 \mathrm{G} \Omega$ & variable & $1: 1$ or $100: 1$ \\
\hline $\mathrm{C} 1198$ & $100 \mathrm{G} \Omega$ & variable & $1: 1$ or $100: 1$ \\
\hline \hline
\end{tabular}

\section{NIST High Resistance TRANSFER StANDARDS}

Presently NIST has nine transfer standards used in the high-resistance laboratory, covering the decade ranges from $10 \mathrm{k} \Omega$ to $100 \mathrm{G} \Omega$. Five of the nine standards are internally guarded, two are externally guarded at the coaxial shields of each main resistor junction, and the remaining two can be externally guarded at the end terminations. The different nominal values of the internal guard resistors make it impossible to take full advantage of the internal guarding for all scaling measurements due to different main-to-guard ratios. Table I lists the main and guard resistors for the nine transfer standards. All of the internally guarded transfer standards except for J/895 were built in the late 1960's at NIST. The two newest transfer standards, C1098 and C1198, use external guard networks and do not have internal guard networks at the present time. Internal guard networks providing guarded transfer standards C1098 and C1198 main-to-guard ratios of 100:1 are expected to be added, allowing routine guarded comparison measurements of $\mathrm{C} 1098$ and $\mathrm{C} 1198$ against the wirewound $1 \mathrm{G} \Omega$ transfer standard $\mathrm{J} / 9$. Transfer standards $\mathrm{J} / 895, \mathrm{C} 1098$, and $\mathrm{C} 1198$ all have coaxial terminations at each resistor junction, permitting quick connection of external guard networks and fixtures for series-parallel and parallel connections for the transfer standards.

Transfer standards $\mathrm{J} / 6, \mathrm{~J} / 7$, and $\mathrm{J} / 895$ have a main-to-guard ratio of $1: 1$ and transfer standards $\mathrm{J} / 8$ and $\mathrm{J} / 9$ have a mainto-guard ratio of 100:1. The choices of guard resistors were determined by several factors that made it difficult to build all of the transfer standards with the same main-to-guard ratio. For example, in the case of $\mathrm{J} / 6$, if the guard resistors were $1 / 100$ of the value of the main resistors, as in $J / 8$, this would form a guard resistance of $100 \Omega$ when $\mathrm{J} / 6$ is in the parallel configuration. A $100 \Omega$ guard resistance would draw excessive current at test voltages above $5 \mathrm{~V}$, overloading the current capacity of the bridge and causing self-heating of the transfer standard because of the excessive heat being dissipated by the guard resistors. $\mathrm{J} / 8$ and $\mathrm{J} / 9$ were constructed using guard resistors $1 / 100$ of the value of the main resistors to maintain accurate guard potentials throughout the transfer standard. Additionally, $1 \mathrm{G} \Omega$ guard resistors needed to match guard

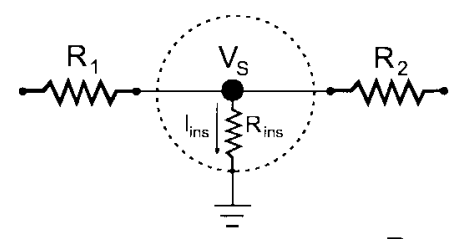

(a)

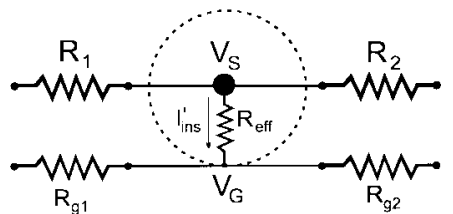

(b)

Fig. 2. Unguarded main resistor junction shown in (a), leakage current $I_{\text {ins }}$ flowing to ground. In (b), the guard resistors drive potential $V_{G}$ at nearly the same potential as the main resistor junction potential $V_{S}$ limiting the leakage current $I_{\text {ins }}^{\prime}$ flowing through the insulator $R_{\text {ins }}$.

potentials within $1 \%$ of the main resistor potentials may not have been readily available when $\mathrm{J} / 8$ and $\mathrm{J} / 9$ were built.

\section{GuARD CIRCUIT THEORY}

The guard resistors are used to effectively increase the resistance of the insulation, $R_{\text {ins }}$, between the main and guard networks. Without a guard network, $R_{\text {ins }}$ can be expressed as

$$
R_{\mathrm{ins}}=V_{S} / I_{\mathrm{ins}}
$$

where $I_{\text {ins }}$ is the current flowing from a main resistor potential, $V_{S}$, to ground potential as shown in Fig. 2(a). Fig. 2(b) shows a guarded network with a current $I_{\text {ins }}^{\prime}$ flowing through the insulator that separates the main and guard resistors. The current $I_{\mathrm{ins}}^{\prime}$ is defined as

$$
I_{\mathrm{ins}}^{\prime}=\left(V_{S}-V_{G}\right) / R_{\mathrm{ins}}
$$

where $V_{S}$ and $V_{G}$ are the standard and guard resistor potentials.

By substituting the guarded insulator current in (2) into the insulator current of (1), it can be shown that the effective insulation resistance is

$$
R_{\mathrm{eff}}=R_{\mathrm{ins}} * V_{S} /\left(V_{S}-V_{G}\right) .
$$

Assuming the guard resistors can match $V_{G}$ within $1 \%$ of $V_{S}$, the guarding will increase the effective resistance of the insulator by 100 times the actual resistance of the insulator.

\section{EXTERnal GuARD NeTWORKS}

Due to the different main-to-guard ratios of NIST transfer standards, it is not always possible to compare two transfer standards using their internal guard networks. In this situation, an external guard resistor is connected across the terminals of one transfer standard isolated from the internal guard network. This leaves the internal main resistor junctions unguarded. In the new design, the interchangeable external guard modules provide complete guarding and eliminate the need to disconnect the internal guard networks. The external guard network is attached in parallel to the internal guard network, thereby decreasing the main-to-guard ratio and placing the coaxial shields at the same potential as the standard resistor junctions. A transfer standard like $\mathrm{J} / 895$ could have either a $1: 1$ or a 1:100 


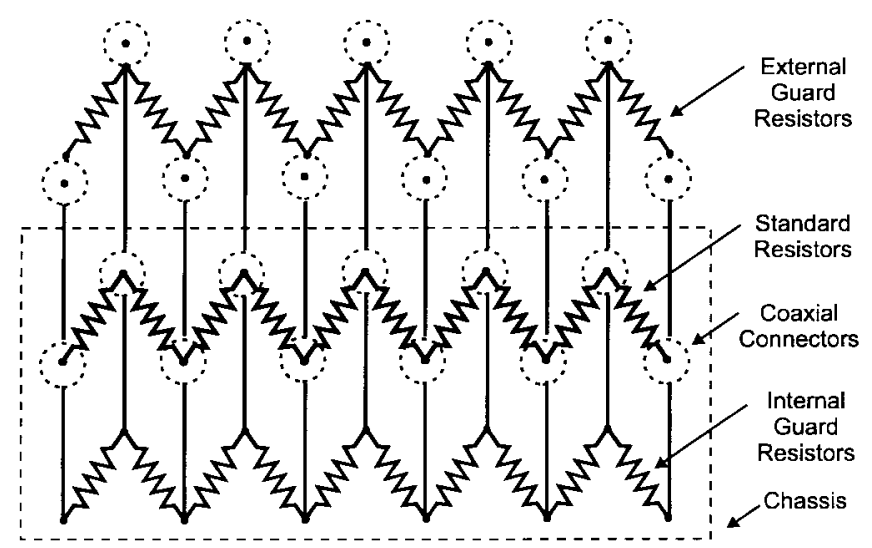

Fig. 3. Guarded transfer standard shown with internal and external guard resistors. Guarded transfer standard can have a main-to-guard ratio of 1:1 with internal and external guard networks connected in parallel. Removal of the external guard network creates a main-to-guard ratio of 100:1.

main-to-guard ratio permitting fully guarded comparisons with other transfer standards.

The improved design uses interchangeable external guard modules so that several values of guard networks can be selected allowing different main-to-guard ratios. The external guard networks can be used with or without an internal guard network to allow accurate guard potentials to be generated at the coaxial shield of each standard resistor junction. Fig. 3 shows a guarded transfer standard with both internal and external guard resistors. Typically the parallel combination of internal and external guard resistors would be no larger than $1 / 100$ of the value of the internal guard resistors, giving the paralleled guard networks a nominal guard resistance within $1 \%$ of the external guard network's nominal resistance.

To test the concurrent use of internal and external guard networks, NIST transfer standard J/895 was calibrated by NIST transfer standard $\mathrm{J} / 7$ for a 10:1 bridge ratio using only the internal guard networks. Both transfer standards have a main-to-guard ratio of $1: 1$ and were in series mode. This permits calibration of $\mathrm{J} / 895$ by $\mathrm{J} / 7$ using the internal guard networks of both transfer standards. An external guard network was then added to $\mathrm{J} / 895$ making the standard-to-guard ratio $100: 1$, allowing the use of transfer standard $\mathrm{J} / 8$ to calibrate $\mathrm{J} / 895$ for a 1:1 bridge ratio in series mode with all resistor junctions guarded. The calibrations of $\mathrm{J} / 895$ by both standards $\mathrm{J} / 7$ and $\mathrm{J} / 8$ differed by less than the Type A relative standard uncertainty of $0.2 \times 10^{-6}$ for both types of measurements which demonstrates that external guard networks can be used to fully guard all resistor junctions when comparing transfer standards having different main-to-guard ratios.

\section{Evaluation of Guard Circuit EFFEctiveness}

To test the effectiveness of the guard circuit, external "leakage" resistors were used to provide a relatively low-resistance path for leakage current to flow between the guard and main resistor networks, thereby simulating a connector having poor or contaminated insulation. Polytetrafluoroethylene (PTFE) insulated connectors typically have resistances on the order of $10^{12} \Omega$ to $10^{14} \Omega$ and above, so leakage resistors $\left(R_{\mathrm{LEAK}}\right)$

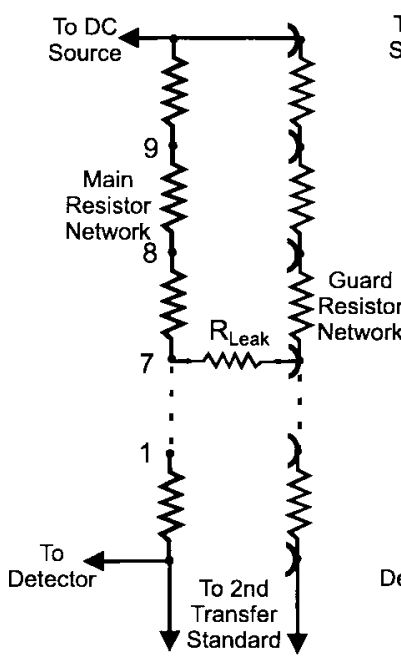

(a)

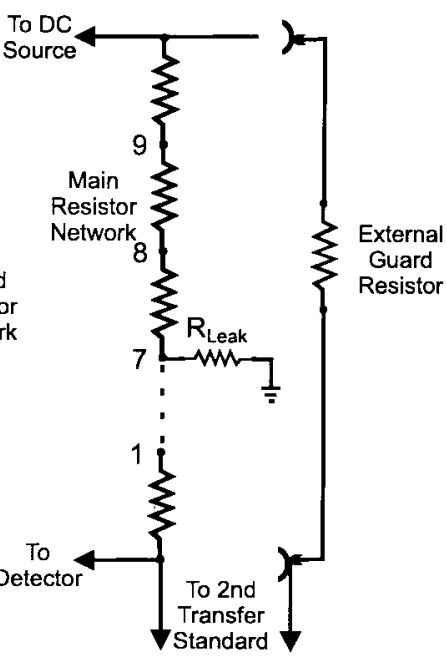

(b)
Fig. 4. Leakage resistance $R_{\mathrm{LEAK}}$ connected to the transfer standard to test the effectiveness of a guard network. $R_{\mathrm{LEAK}}$ connected between the main and guard networks in (a). Leakage currents to ground are impeded by the guard network. In (b), the guard network has been removed allowing leakage currents to flow to ground. An external guard resistor maintains guard potential at main resistor network terminations.

ranging from $1 \mathrm{G} \Omega$ to $100 \mathrm{~T} \Omega$ were used for these tests. Measurements were made with the guard network energized and then repeated with the guard network disabled. The guard network was disabled by removing the external guard network and isolating the internal guard network from the guarded Wheatstone bridge or active-arm bridge. The isolated internal guard network was held at ground potential. Fig. 4(a) shows how the leakage resistors were connected to the transfer standards with the guard network energized. Fig. 4(b) shows the guard network disabled and a single external guard resistor driving the end termination shields of the transfer standard at guard potentials, allowing the transfer standard to be measured with the guarded resistance bridges. Without a guard circuit in place, leakage currents are able to flow between the unguarded transfer standard and ground. Resistor junctions are numbered from one to nine with one being closest to the detector and nine being closest to the low-impedance dc source. Connections to the dc source, detector, and standard and guard resistors in the bridge circuit are also shown.

Figs. 5-7 show guarded and unguarded measurements made on the $100 \mathrm{M} \Omega$ transfer standard J/895. The effects of changing leakage and guard resistors could be readily detected and observed at the $100 \mathrm{M} \Omega$ level where wirewound standards are well characterized and noise levels are orders of magnitude smaller than at the $100 \mathrm{G} \Omega$ level. Type A relative standard uncertainties for the guarded Wheatstone bridge and activearm bridge are $4 \times 10^{-6}$ and $0.2 \times 10^{-6}$ [2], [3], respectively, at the $100 \mathrm{M} \Omega$ level. The data reported in Figs. 5-7 are relative differences of the transfer standard with $R_{\mathrm{LEAK}}$ connected to the main resistor network. Measurements made with both the Wheatstone bridge and active-arm bridge agreed within the Type A relative standard uncertainty for the Wheatstone bridge; verifying that the relative differences observed were independent of the measurement system. 


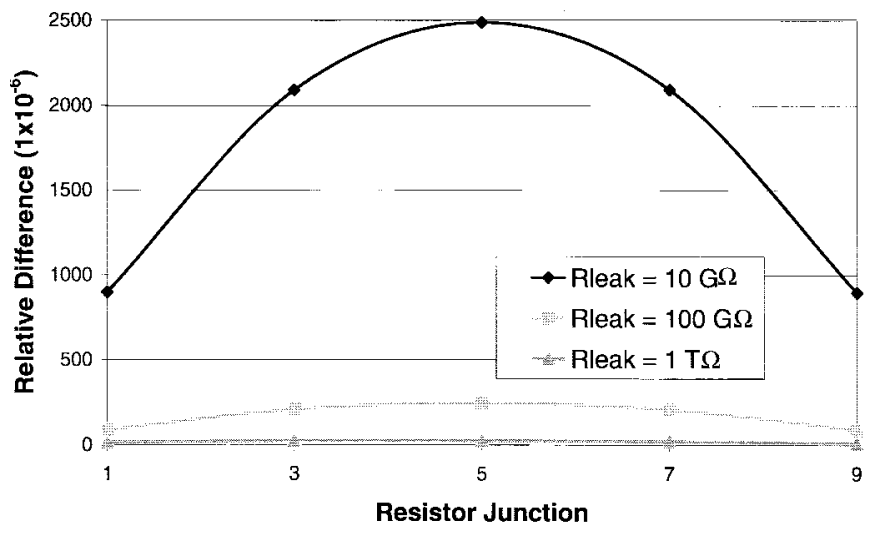

Fig. 5. Relative differences for $100 \mathrm{M} \Omega$ transfer standard with resistor junctions unguarded. $10 \mathrm{G} \Omega, 100 \mathrm{G} \Omega$, and $1 \mathrm{~T} \Omega$ leakage resistance $R_{\mathrm{LEAK}}$ applied to resistor junctions of transfer standard J/895 with internal guard isolated.

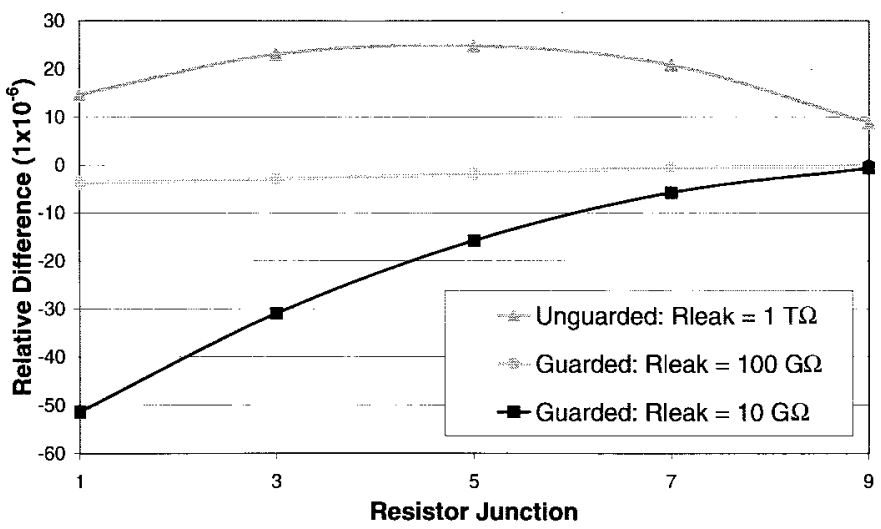

Fig. 6. Relative differences with junctions guarded and unguarded. Leakage applied closest to detector creates greatest deviation for guarded transfer standard with guard network mismatched by $1 \%$.

Fig. 5 shows measurements made on the unguarded 100 $\mathrm{M} \Omega$ transfer standard $\mathrm{J} / 895$ with $R_{\mathrm{LEAK}}$ of nominal values 10 $\mathrm{G} \Omega, 100 \mathrm{G} \Omega$, and $1 \mathrm{~T} \Omega$ connected to the resistor junctions as shown in Fig. 4(b). The measurements were made for bridge ratios of 1:1 and 10:1. Bridge ratio had no significant effect on the leakages observed. As the data in Fig. 5 indicates, leakage resistance can cause significant relative differences of the value of the main resistance network when the guard network is not active. The largest relative differences are when $R_{\mathrm{LEAK}}$ is placed at the midpoint of the transfer standard. The smallest relative differences are at the junctions closest to the low-impedance dc source and the null detector, which are the paths of least resistance to ground. Since the dc source is of low impedance and the detector input is at a virtual ground, the midpoint of the transfer standard is the region most likely to be susceptible to leakage currents to ground. The relative differences are proportional to the parallel resistance of the two halves of the transfer standard at the resistor junction where $R_{\mathrm{LEAK}}$ is connected to ground.

The sequence of measurements was repeated with the guard network energized and $R_{\text {LEAK }}$ connected between the standard and guard networks at the resistor junctions as shown in Fig. 4(a). Fig. 6 shows guarded and unguarded measurements

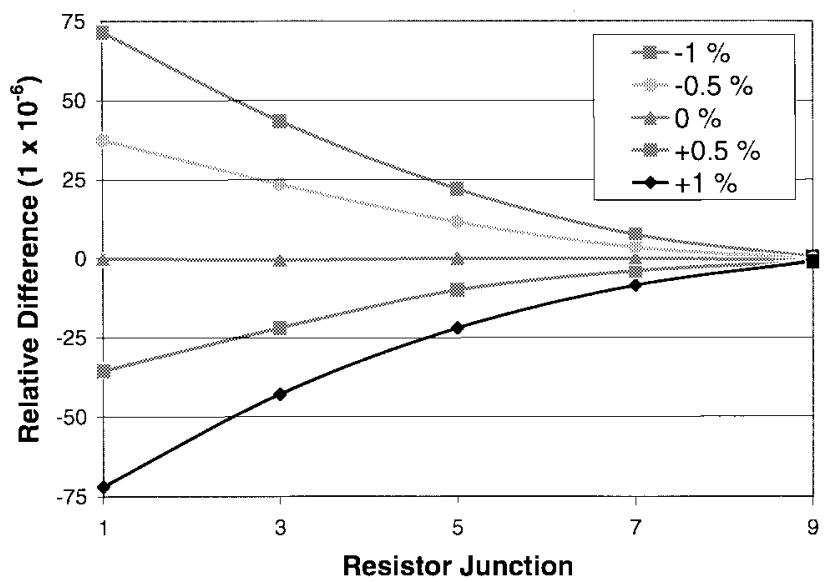

Fig. 7. Relative differences for mismatched guard networks. $1 \mathrm{G} \Omega$ leakage resistance $R_{\text {LEAK }}$ used to test effectiveness of mismatched guard networks. Mismatched guard creates greatest leakage currents near the detector where guard resistor network voltage deviates the most from main resistor network voltage.

for $R_{\mathrm{LEAK}}$ of nominal values $10 \mathrm{G} \Omega, 100 \mathrm{G} \Omega$, and $1 \mathrm{~T} \Omega$. The guarded network with a $R_{\mathrm{LEAK}}$ of $100 \mathrm{G} \Omega$ reduced relative differences caused by $R_{\mathrm{LEAK}}$ to approximately $1 \%$ of the relative difference when the guard network was inactive. However, guarded measurements made using $R_{\mathrm{LEAK}}$ equal to $10 \mathrm{G} \Omega$ showed a disturbing increase in the relative difference as $R_{\mathrm{LEAK}}$ was connected to junctions closer to the detector. Further investigation revealed that a mismatch between the main and guard networks generated excessive leakage currents which were the source of the difference. This mismatch was caused by paralleling the internal $100 \mathrm{M} \Omega$ guard network of $\mathrm{J} / 895$ with an external guard network of $1 \mathrm{M} \Omega$ creating a combined guard network of approximately $99 \mathrm{k} \Omega$. An external guard network of $1.01 \mathrm{M} \Omega$ would create a combined guard network of $1 \mathrm{M} \Omega$ thereby properly guarding the transfer standard from leakage currents near the detector. All of NIST's new external guard networks will be constructed to have the correct main-to-guard ratio.

Fig. 7 shows the effect of a guard mismatch of $\pm 1 \%$ of the total resistance of the transfer standard coupled with $R_{\mathrm{LEAK}}$ of $1 \mathrm{G} \Omega$. This combination of conditions causes excessive leakage current to flow near the detector. The $100 \mathrm{M} \Omega$ transfer standard would not be routinely subject to leakages less than $1 \mathrm{~T} \Omega$ so matching the guard network within $1 \%$ of the main network is not as critical as it would be for a $100 \mathrm{G} \Omega$ transfer standard. A mismatched guard network coupled with the effect of leakage resistance can create large relative differences in the measured value of a transfer standard. Mismatched guard voltages at the detector can be prevented by careful matching of guard resistors to main resistors during construction of transfer standards.

Some film-type resistors have large corrections from nominal that would make selecting a well matched guard resistor difficult especially for unknown customer resistors in the range $100 \mathrm{G} \Omega$ to $100 \mathrm{~T} \Omega$. A separate dc source for the guard of the unknown resistor can be used to reduce leakage currents near the detector by adjusting the voltage applied to the customer guard resistor. The guard potential of the coaxial shields of the 
transfer standard could then be matched to the potentials of the resistor junctions without having to select a guard resistor to closely match an unknown customer resistor that may have a large correction from nominal.

\section{SUMMARY}

By using internal and external guard networks for highresistance transfer standards, complete guarding can be used to reduce leakage currents to ground for many bridge ratios, allowing the comparison of transfer standards that are fully guarded at all resistor junctions over the range $10 \mathrm{M} \Omega$ to $100 \mathrm{~T} \Omega$. External guard networks have been constructed and tested and can be used alone or in parallel with an internal guard network to reduce the effect of leakage currents flowing from the measurement circuit to ground. When external guard networks are used in parallel with internal guard networks, the parallel resistance should be well matched to the nominal ratio of the transfer standard. Two guarded transfer standards at the $10 \mathrm{G} \Omega$ and $100 \mathrm{G} \Omega$ decade levels have been constructed at NIST using external guard networks. The insertion of a leakage resistance in parallel with the insulation of guarded and unguarded transfer standards has been used to demonstrate the effectiveness of guard networks to protect transfer standards from unwanted leakage currents to ground.

Further investigation of guarding techniques and guard networks is planned along with the construction of additional transfer standards at and above the $1 \mathrm{~T} \Omega$ level. These new transfer standards will use the resistor mounting techniques developed at NIST, including the evaluation of a hermetically sealed canister that permits guarding of the resistor element at both terminations of the resistor element.

\section{ACKNOWLEDGMENT}

The author recognizes coworkers R. Dziuba and L. Scott who have heat-treated, assembled, and hermetically sealed the resistor elements used to construct the new transfer Standards C1098 and C1198.

\section{REFERENCES}

[1] B. V. Hamon, "A 1-100 $\Omega$ build-up resistor for calibration of standard resistors," J. Sci. Instrum., vol. 31, pp. 450-453, Dec. 1954.

[2] R. F. Dziuba, P. A. Boynton, R. E. Elmquist, D. G. Jarrett, T. P. Moore, and J. D. Neal, "NIST measurement services for dc standard resistors," Nat. Inst. Stand. Technol. Tech. Note 1298, pp. 30-44, 1992.

[3] D. G. Jarrett, "Automated guarded bridge for calibration of multimegohm standard resistors from $10 \mathrm{M} \Omega$ to $1 \mathrm{~T} \Omega$," IEEE Trans. Instrum. Meas., vol. 46, no. 2, pp. 325-328, Apr. 1997.

[4] R. F. Dziuba, D. G. Jarrett, L. L. Scott, and A. J. Secula, "Fabrication of high-value standard resistors," in CPEM'98 Conf. Dig., Washington, DC, July 1998, pp. 309-310.

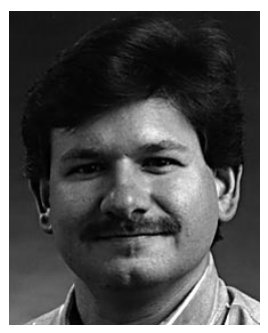

Dean G. Jarrett (S'88-M'90-SM'99) was born in Baltimore, MD, in 1967. He received the B.S. degree in electrical engineering from the University of Maryland, College Park, in 1990, and the M.S degree in electrical engineering from The Johns Hopkins University, Baltimore, in 1995.

$\mathrm{He}$ began working at the National Institute of Standards and Technology (NIST), Gaitherburg, MD, in 1986 as a Cooperative Education Student from the University of Maryland. During this time, he worked in the dc resistance area on the automation of resistance calibration systems. In 1991, he joined NIST fulltime as an Electrical Engineer working on the development of an automated ac resistance calibration system and the development of new resistance standards. Since 1994, he has worked in the High Resistance Laboratory developing automated measurement systems and improved standard resistors to support standard resistor calibration services from $1 \mathrm{M} \Omega$ to $100 \mathrm{~T} \Omega$. 